\title{
The Effect of Flow Pattern on Combustion and Pollution Generation in a Jet Engine \\ Hossein Keshtkar*
}

Khaje Nasir University of Technology, KNTU, Tehran, Iran

\begin{abstract}
As we know internal combustion engines are not limited to reciprocating engines only, engines such as gas turbines are also considered to be internal combustion engines. A gas turbine generally consists of a compressor in upstream of the flow and a turbine at downstream of the flow which have been coupled to rotate together, with a combustion chamber located between these two sections. The operation of a gas turbine engine is similar to that of a power generation steam turbine, except in the former case hot steam is replaced by air entering the engine. The atmospheric air initially enters the compressor, in which it experiences significant compression and pressure buildup. The compressed air is guided to the combustion chamber, in which by injecting fuel and igniting the mixture, combustion is achieved with significant thermal energy being released. The energy released causes combustion products to expand and accelerate towards the turbines located downstream. The flow of these gases through the turbine generates power to run the compressor as well as to provide auxiliary power for other purposes as well as to generate thrust for the engine. The application of the gas turbine determines whether it's designed to achieve optimum thrust power, or to gain maximum power generation. For a stable combustion performance with high efficiency the design of the combustion chamber need to be so that it insures an adequate mixture of air and fuel and also provide sufficient reaction time for fuel ignition. Because of the operational conditions of a jet engine, incoming air will enter the combustion chamber with extensive velocity. This air speed can significantly reduce the combustion quality, or even transport the flame downstream blowing out the combustion entirely. Thus a recirculation zone needs to be provided, to both reduce incoming air speed and create a uniform temperature field within the combustion region. A uniform temperature field can prevent generation of cold or hotspots within combustion region which can greatly affect the produced pollutants related to engine combustion.
\end{abstract}

\section{Keywords: Velocity; Pollution; Jet; Engine}

\section{Introduction}

An ideal gas turbine follows by the Brighton cycle, undergoing three thermodynamic phases namely; isentropic compression, isopressure combustion and finally an isentropic expansion. However in an actual gas turbine, deviations are expected from the ideal Brighton cycle, such as energy loss due to friction based irreversibility's following the compression stage in both axial and centrifugal compressors. A jet engine (Figure 1) performs similarly to a gas engine, except its main purpose is to provide thrust power alongside some shaft rotation inertia, powering the compressor (Figures 1 and 2). The compressor unit,

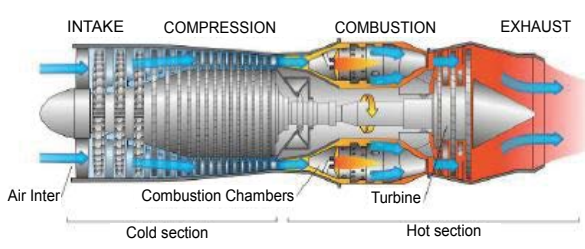

Figure 1: Visual demonstration of a jet engine.

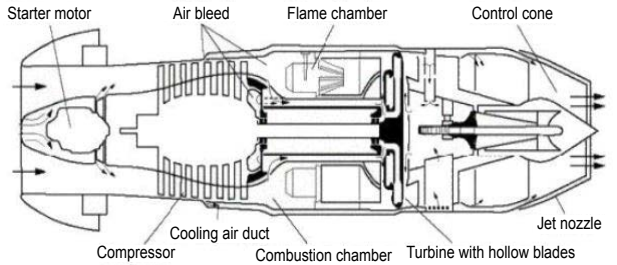

Figure 2: Demonstration of important components within a jet engine. which is fed by the power collected in the unit's turbine, pressurizes the atmospheric air in the combustion chamber, where it's mixed with injecting fuel and ignited. The high speed exhaust gasses discharging to the atmosphere generates the thrust, propelling the engine. The exact performance of the engine depends on its application and usage.

One of the recent advancements of gas turbines is achieving homogenous spread combustion within the combustion chamber. To achieve homogenous combustion, one needs to maintain a uniform temperature field within the chamber which reduces the chance of building hotspots, promoting thermal NOx production. Homogeneous combustion is achieved by using premixed, partly premixed or even non-premixed fuel using high turbulence to develop a uniform mixture of air and fuel prior to combustion. Swirling flames have a wide spread application in providing a recirculation zone which effectively promoted mixing whilst promoting to maintain a stable flame.

\section{Materials and Methods}

\section{Combustion chamber}

The main chamber in which fuel is sprayed using multi-nozzleinjection sprays onto the incoming air is referred to as the combustion

*Corresponding author: Hossein Keshtkar, Khaje Nasir University of Technology, KNTU, Tehran, Iran, Tel: +98218888 2991; E-mail: hkeshtkar_mech@email.kntu.ac.ir

Received May 24, 2018; Accepted June 19, 2018; Published June 26, 2018

Citation: Keshtkar H (2018) The Effect of Flow Pattern on Combustion and Pollution Generation in a Jet Engine. J Astrophys Aerospace Technol 6: 157. doi:10.4172/2329-6542.1000157

Copyright: (C) 2018 Keshtkar H. This is an open-access article distributed under the terms of the Creative Commons Attribution License, which permits unrestricted use, distribution, and reproduction in any medium, provided the original author and source are credited. 
chamber. By sudden release in thermal energy, the combustion products are accelerated and expanded almost spontaneously. The sudden expanding gasses flow through smooth streamlines with a uniform temperature distribution which provides enough energy to rotate the turbine blades. This procedure has to be accomplished of minimal pressure drop and optimum energy release rate considering the limited space within the turbine unit. Although the released energy is directly proportional to the amount of fuel being injected, but its constrained by the thermal resistance of the materials used. Usually the chamber pressure is required to achieve no more than $170^{\circ} \mathrm{C}$ for safety and has to be maintained above $70^{\circ} \mathrm{C}$ to prevent flame blowout. It's important to point out the pressurized compressed air inside the chamber will be achieve temperature of about $20^{\circ} \mathrm{C}$ to $55^{\circ} \mathrm{C}$ even prior to combustion, just because of the compression effects.

Air entering the combustion chamber of a jet engine usually has a mean velocity of some $150 \mathrm{~m} / \mathrm{s}$ in operational conditions. This air velocity is extremely high and can cause instant flame blowout, thus it needs to be slowed significantly. To do so a diffuser unit is used, which can slow down the air feed to about $25 \mathrm{~m} / \mathrm{s}$. But the flame velocity of regular jet fuel usually does not exceed a few meters per second. Any flow speed above this can carry the flame downstream, blowing out the combustion. Therefore the air velocity needs to be further reduced. This is achieved through creating a low axial velocity region within the chamber to maintain a stable flame condition. Also if the entire air volume passing through the compressor enters the combustion chamber, fuel to air ratios of 1:45 up to 1:130 will be generated, which will also vary throughout the chamber which is much higher than that required by most applicable fuels which require an $\mathrm{A} / \mathrm{F}$ ratio of $1: 15$ to $1: 25$ in order to achieve optimum efficiency. To do so, we must separate a small amount of the entire air flow towards the chamber using combustion liners. The flow mass is carefully measured to attain optimum combustion conditions. Located immediately after the combustion liners, are the swirl vanes followed by perforated flare which guide the flow towards the chamber. The flow spin generated causes an induced upstream flow and a recirculation zone, which both help to achieve a uniform mixture as well as to reduce flow velocity to acceptable speeds. A portion of the remaining air flow which doesn't enter the combustion liners flows in between these tubes and the main engine body, assisting to cool down the engine under heavy performance conditions.

\section{Parameters effecting combustion}

Amongst different parameters governing the combustions, one can refer to the flow patterns within combustion chamber, its residence time inside the chamber, chamber geometry and the type of fuel burnt. By increasing gas residence time inside the chamber and preventing local quenching, $\mathrm{CO}$ and unburnt hydro carbons will have enough time to completely participate in the reactions producing $\mathrm{CO}_{2}$ and other safe combustion products. This is because $\mathrm{CO}$ burn much slower that other radicals, therefore some $4 \mathrm{~ms}$ time is needed to ensure complete burning of carbon monoxide. As can be seen from Figure 3, we cannot effectively prevent $\mathrm{CO}$ forming if the combustion temperature drops below $110^{\circ} \mathrm{C}$. This graph was produced based on time required to maintain exhaust CO levels at 10ppm in a commercial gas turbine [1].

Also in order to achieve a stable stationary flame, we need to take a deeper look into the combustion velocity. As we know, the flame travels through the unburnt mixture with a certain speed. Thus the velocity of the incoming air/fuel mixture must be in accordance with the flame speed; otherwise it may separate the flame from its location, transporting it downstream causing a blowout in the original

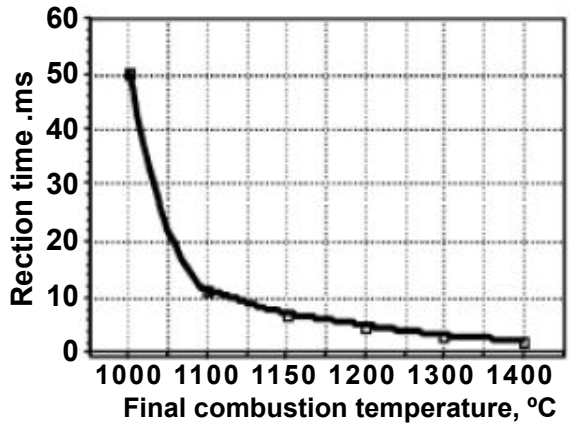

Figure 3: The effect of reaction time on final combustion temperature of products [1].

flame location. If by chance the oncoming flow velocity is lower than that of the flame combustion velocity, the flame may start to travel upstream through the unburnt mixture; which also could lead to an unstable combustion. Thus to prevent these problems, one needs to design the combustion chamber so that a recirculation of combusted gases occur, maintaining the flame at its designated location. These recirculation zones need to have a velocity no more than that of the flame, and also tend to travel slightly the upstream to ensure a uniform mixture is achieved and minimum unburnt gasses escape. The swirling effect caused by the recirculation zones prevent the flame travelling downstream, and also provides it with sufficient time to ensure optimum combustion. The flow development downstream of the recirculation zone is mainly due to centrifugal force created by the swirling motion. This centrifugal force develops a low local pressure buildup within the combustion chamber. From a certain point beyond, the centrifugal force is dampened by viscous effects and the high pressure surrounding regions cause a vortex breakdown ending the circulating motion of the flow [2].

\section{Types of flow pattern inside combustion chamber}

Several different types of flow patterns can co-exist in the combustion chamber, having the role of ensuring a sufficient air/ fuel mixture, a uniform temperature distribution and stabilizing the flame. As mentioned before, by generating recirculation zones we can promote the air/fuel mixture quality and keep combustion within stable conditions. The recirculation zones themselves are generated by the centrifugal effect of swirls and vortexes induced by different flow patterns. The size of the recirculation zone can vary with the size of the vortexes as well as the combustion chamber geometry. Extensive research has been conducted that prove swirls intensity can significantly affect flame modes [3,4].

The swirl intensity, or the degree of a swirl, which characterize the swirl number is shown by ' $\mathrm{S}$ '. Swirl intensity is a dimensionless parameter showing the ratio of flow's tangential momentum flux versus its axial momentum flux.

$$
\mathrm{S}=\frac{2 \mathrm{G}_{\grave{\mathrm{e}}}}{\mathrm{RG}_{\mathrm{x}}}
$$

In which $G_{\theta}$ represents the axial flux of the tangential momentum and $G_{x}$ stands for axial flux of the axial momentum, and $\mathrm{R}$ is the outer radius of the annulus. We can also express both momentum fluxes as:

$$
\begin{aligned}
& G_{\theta}=\int_{0}^{R} \rho(W r) U 2 \pi r d r \\
& G_{x}=\int_{0}^{R} \rho U^{2} 2 \pi r d r
\end{aligned}
$$


Where $\mathrm{U}, \mathrm{W}$ and $\rho$ are axial velocity, tangential velocity and density respectively. It's also interesting to know, in a free jet stream, both $G_{x}$ and $G_{\theta}$ are constants and are independent of the actual flow.

We can classify swirls intensity into two groups, namely "low swirl flow" ( $S \leq 0.4)$ and "high swirl flow" $(S \geq 0.6)$ plus a transient swirl region in between these groups which is usually neglected as a separate class [5].

Low swirl flow: Generally in this type of flow, flow pattern is not affected by swirl velocity as is displayed in Figure 4 . This type of swirl generates a lateral pressure gradient inside the flow. Compared with a non-swirling flow, low swirling flows are wider and slower. Any attempts of producing a flame with low swirl flow faces practical restrictions as the flame will be unstable in this situation. None the less, modeling low swirl flows remains very important in characterizing flow patterns. There exist applications in which low swirl flow may potentially affect flame structure, generating a recirculation zone, such as in fire whirls, in which swirl plays an effective role in lengthening the flame.

High swirl flow: In high swirling flows, pressure gradients are sufficiently large enough to produce central toroidal recirculation zones which don't exist in low swirling flows as can be seen from Figure 5.

As can be seen from Figures 2 and 3, high intensity swirls can generate longitudinal flow patterns. Also compared with a non-swirling flow, high swirl flows potentially have a wider dimension and a much slower axial velocity, and also usually contain a central recirculation zone. As we know, recirculation zones play an important role in flame stability, reducing a flow's axial velocity providing sufficient time for complete combustion to take place. Whilst also promoting uniform fuel/air mixture and preventing any cold spots taking place.

\section{The effects of swirl on combustion pollution}

Multiple researches have been conducted using ANSYS (CFD) which correlates the production of $\mathrm{NO}$ and $\mathrm{CO}$ pollutants in the

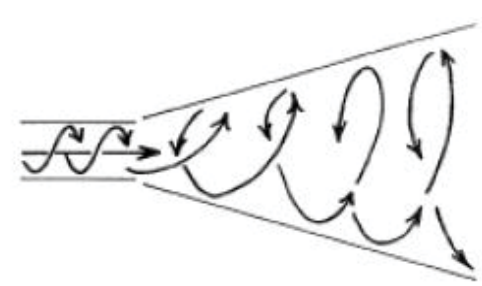

Figure 4: Low swirl flow $(\mathrm{S}<0.4)$ which produces lateral flow alongside pressure gradients.

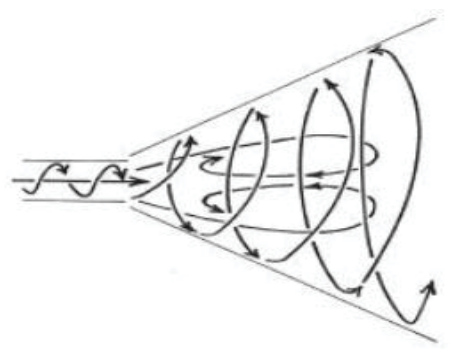

Figure 5: High swirl flow $(S>0.6)$ which generates larger lateral flow zones with greater pressure gradients.

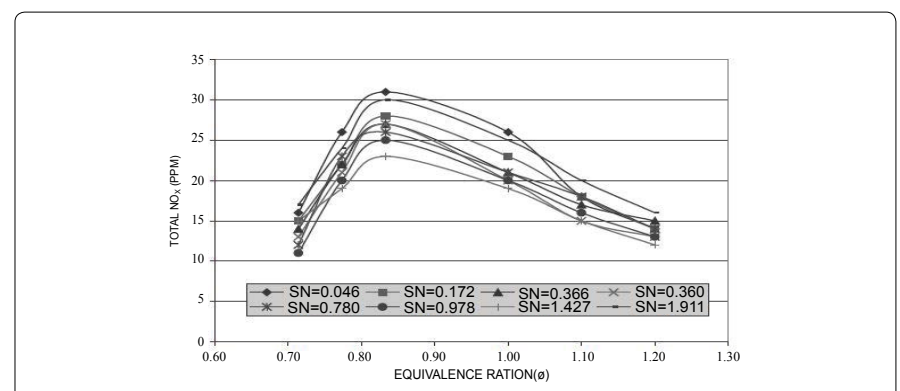

Figure 6: Nitrogen Oxide (NOx) generated under the effect of different swirl intensities and various equivalence ratio [6]

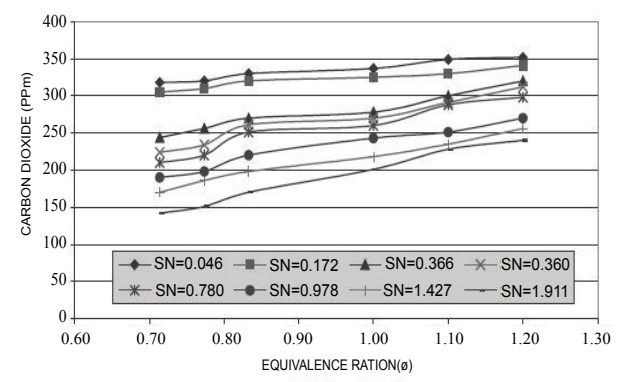

Figure 7: Carbon Monoxide (CO) generated under the effect of different swirl intensities and various equivalence ratio [6]

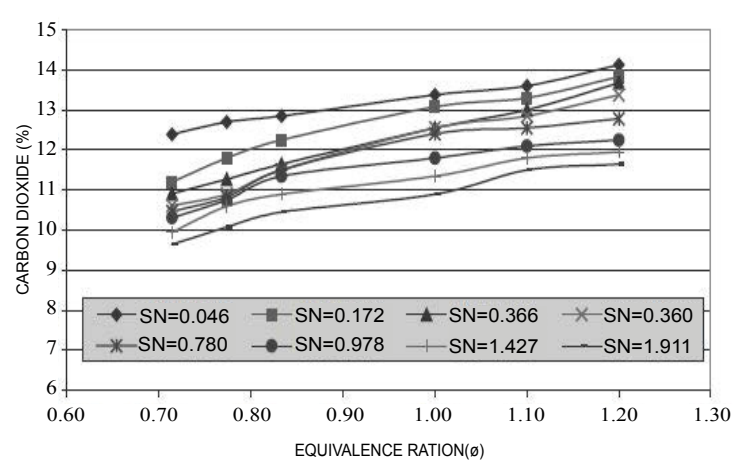

Figure 8: Carbon Dioxide $\left(\mathrm{CO}_{2}\right)$ generated under the effect of different swirl intensities and various equivalence ratio [6].

combustion throat region with the swirl intensity of the flow within that region. Higher swirl intensities produce wider but shorter flames, they also assist in reducing peak maximum temperature, eliminating hot spots and preventing the formation of NOx. High intensity swirls also generate an optimized uniform temperature distribution within the combustion region [6-9].

\section{Results and Discussion}

Jaafar et al. simulated the generation of $\mathrm{CO}$ and $\mathrm{NOx}$ under the effect of various swirl intensities [10]. They used swirler vanes of $10^{\circ}, 20^{\circ}, 30^{\circ}, 40^{\circ}, 50^{\circ}, 60^{\circ}$ and $70^{\circ}$ angles to generate swirl intensity. Their research and simulation showed that a vast reduction in oxides of nitrogen (NOX) emissions is achieved when the vane angle was increased from $10^{\circ}$ to $60^{\circ}$ (SN 0.046 to SN 1.427) (Figure 6).

This was true for the entire range of operating equivalence ratios considered. Although emission levels lower than 35 ppm NOx was 
reported for all operating equivalent rations, for swirl number of 1.427, NOX emissions reduction of about 26 percent was obtained at equivalence ratio of 0.83 compared to the swirl number of 0.046 at the same equivalence ratio.

This goes to prove that swirl significantly helps the mixing of fuel and air prior to ignition and is also signified by a reduction in NOx emissions. However, a turning point occurs at swirl number of 1.911 in this experiment, in which displays that NOx emissions seem to increase compared with other swirl number data for a specific equivalency ratio; in fact NOx increase a mere 23 percent compared with a swirl number of 1.427 at equivalency ratio of 0.83 . This can be explained by the opposing effects of using a very high swirl number in the combustion chamber; from which we can point out to a reduction in combustion stability along with an increase of emissions at very high swirl numbers.

Figure 7 shows carbon monoxide (CO) emissions versus equivalence ratio for all swirl numbers considered in the simulation. There was a 33 percent, 40 percent and 48 percent reduction in carbon monoxide (CO) emission for swirl number 0.978, 1.427 and 1.911 respectively compared to swirl number of 0.046 at the equivalence ratio of 0.833 . The concentration of carbon monoxide emission increases with increase in the equivalence ratio. This was anticipated due to the fact that any measure of decreasing NOx will tend to increase CO since both emissions are on the different side of the balance [11].

As we can see, the concentration of carbon monoxide emissions increases even for the case with swirl number of 1.911. As explained, we expected CO and NOx to be on opposite sides of the balance, and to experience an increase in one side when the opposing side reduces. This is not the case for the swirl number 1.911 here; as we can see we are facing both an increase in NOx emissions alongside an increase in carbon monoxide concertation. This can be blamed upon the unstable combustion conditions caused by such a high swirl number, which as explained before, could turn against our favor and increase toxic emissions rather than being helpful [12-16].

Also we noticed that the increase was higher than expected, which indicates that some fuel escaped unburned, which was the product of incomplete combustion. Figure 8 shows a plot of carbon dioxide $\left(\mathrm{CO}_{2}\right)$ emissions versus equivalence ratio for all flow swirls considered. There was a slight decrease in carbon dioxide emissions by increasing the swirl number. This was seen throughout the entire range of operating equivalence ratios in the simulation. There was an 11.6 percent, 11.7 percent and 15.5 percent reduction in carbon dioxide emission for swirl number of $0.978,1.427$ and 1.911 respectively compared to swirl number of 0.046 at the equivalence ratio of 0.833 . Although the decrease was very insignificant compared to the reduction of NOX emissions that was obtained, however as we know the increase of carbon dioxide emissions does not contribute to health problems, as carbon dioxide is more stable and non-toxic. Although, $\mathrm{CO}_{2}$ is a greenhouse gas and can contribute to global climate change, it's not considered is combustion pollution.

\section{Conclusion}

We arrive at a conclusion that flow pattern inside combustion chamber can significantly affect the quality, stability and efficiency of combustion as well as govern the intensity of pollutants generated by combustion. We come to an understanding that recirculation zones help develop a stable flame, whilst giving fuel sufficient reaction time for effective combustion. Recirculation zones promote a uniform temperature distribution within the region, preventing any hotspots or cold spots which both can be hazardous considering combustion efficiency and production of toxic emissions also by employing recirculation zones, we effectively slowdown air flow to adequate amplitude to prevent the transportation and blow-off of the flame. Swirling flow inside the chamber is an effective and well-practiced method of generating recirculation zones. We classified flow swirls in to main categories, consisting of high swirl flow and low swirl flow and explained the definition and difference between these two main groups. We also showed the effectiveness of swirl induced flows on pollution generation of combustion. We studied the amounts of $\mathrm{CO}$, $\mathrm{NOx}$ and $\mathrm{CO}_{2}$ production for different swirl intensities and proved by increasing the swirl number, we could effectively decrease the amount of pollutants released by combustion.

\section{References}

1. Abdel-Gayed HM, Abdel-Ghaffar WA, Shorbagy KE (2003) Main flow characteristic in a lean premixed stabilized gas turbine combustor - Numerica Computations Alexandria University Publications, Alexandria, Greece. 4: 123-136.

2. Lefebvre AH (1999) Gas turbine combustion. Thermal Sciences and Propulsion Center.

3. Charles RE, Emdee JL, Muzio LJ, Samuelsen GS (1988) The effect of inlet conditions on the performance and flow field structure of a non-premixed swirlstabilized distributed reaction. $21^{\text {st }}$ International Symposium on combustion, 3-8 August 1986, Technical University of Munich West Germany 21: 1-2046.

4. Vanoverberghe KP, Den Bulck EVV, Mark JT (2003) Confined Annular swirling jet combustion. Combustion Science and Technology. Taylor and Francis. 175 545-578.

5. Eldrainy YA, Ahmad Ibrahim MFB, Mohd Jaafar MN (2009) Investigation of Radial Swirler effect on flow pattern inside a gas turbine combustor. Canadian Center of Science and Education (CCSE) Journal. 3: 5.

6. Ishak MSA, Jaaafar MNM (2005) The effect of swirl number on reducing emission from liquid fuel burner system. Jurnal Mekanikal. 19: 48-56.

7. Coghe A, Cozzi F, Olivani AA (2005) Experimental analysis of a swirl burner. $8^{\text {th }}$ International conference on Energy for a Clean Environment. Lisbon.

8. Driscoll JF, Chen RH, Youngbin Y (1992) Nitric oxide levels of turbulent jet diffusion flames: Effects of varying residence time and Damkohhler numbe. Combustion and Flame. 88: 37-49.

9. Jaafar MNM, Ishak MSA (2013) The formation of Co-No in a confined can combustor due to swirling flow. Advances in Environmental Science and Energy Planning. pp: 326-342.

10. Ishaka MSA, Jaafarb MNM, Omarb WZM (2015) Experimental Analysis on the Formation of CO-NO-HC in Swirling Flow Combustion Chamber. Jurnal Teknologi. 4: 20-26.

11. Lefebvre $\mathrm{AH}$ (1975) Pollution control in continuous combustion. Fifteenth Symposium (International) on Combustion, 25-31 August 1974, The Toshi Center Hall Tokyo, Japan. The Combustion Institute. 15: 1-1537, 1169-1180.

12. Sravan Kumar P, Punna Rao P (2013) Design and analysis of gas turbine combustion chamber. International Journal of Computational Engineering Research. 3: 36-40.

13. Alarami AM, Elfaghi AM (2014) Optimum design procedures of turboje combustion chamber. $2^{\text {nd }}$ International Conference on Advances in Engineering Sciences and Applied Mathematics (ICAESAM' 2016) Dec. 21-22, 2016 Kuala Lumpur, Malaysia.

14. Grover JH, Kesler MG, Scurlock AC (1957) Preliminary evaluation of a rotating flame stabilizer. Journal of Jet Propulsion. 27: 386-391.

15. Solero G, Coghe A (2014) Effect of injection typology on turbulent homogeneous mixing. Dipartimento di Energetica - Politecnico di Milano. Milan, Italy.

16. Chen RH, Driscoll JF (1988) The role of the recirculation vortex in improving fuel-air mixing within swirling flames. $22^{\text {nd }}$ Symposium on Combustion, Poland. 22: $531-540$ 